\title{
Ultrafast Cellular Automata Dynamics of Phase-change Optical Response
}

\author{
L. Zhang ${ }^{1,2}$, K. F. Macdonald ${ }^{1}$, and N. I. Zheludev ${ }^{1,3}$ \\ 1. Optoelectronics Research Centre and Centre for Photonic Metamaterials, University of Southampton, Southampton, SO17 1BJ, UK \\ 2. School of Mathematics and Physics, Anqing Normal University, Anqing 246133, P. R. China \\ 3. Centre for Disruptive Photonic Technologies, TPI, SPMS, Nanyang Technological University, Singapore 637371 \\ Author e-mail address: kfm@orc.soton.ac.uk
}

\begin{abstract}
We introduce a cellular automata methodology for studying photonics of light-induced phase transitions. Multiphysical complexity over disparate length/timescales is reduced to a simple, heuristic rule/parameter set in a model successfully describing several independent experimental datasets.

(c) 2020 The Authors
\end{abstract}

We present a new methodology for the study of light-induced structural phase changes. These underpin some of the most important emerging 'reconfigurable' photonic technologies but are a complex tangle of processes atomic/molecular structural change, band-structure modification, domain and crystallization dynamics, change of optical properties in inhomogeneous composite media, and transport and dissipation of heat and light - developing simultaneously over length and time scales spanning several orders of magnitude. As such they present significant challenges to both first principles theoretical and numerical modelling approaches. Here, we show for the first time that the problem of describing such complex nonlinear optical processes in phase change materials can be reduced to one of just a few free parameters and evolutionary rules in a classic cellular automata (CA) model.

Here we apply a CA approach for the first time to photo-induced structural transitions and the associated instantaneous nonlinear optical response in a metal. We consider the case of solid gallium near to its bulk melting transition, as a non-trivial system in which optical excitation may proceed via both thermal and non-thermal mechanisms, and surfaces are subject to nanoscale 'pre-melting'. We show that CA can reproduce experimentally measured reflective all-optical switching behaviors over a wide range of fs to ns pulsed optical excitation regimes.

Cellular automata are fully discrete dynamic systems, wherein the state of each cell in a lattice is chosen from a finite set of possible states and each cell evolves in each time step according to a set of transition rules dependent upon its initial state and the those of its neighbors. We consider a two-dimensional CA model of, by design, minimally sufficient complexity to describe the photo-induced metallization dynamics of gallium at a planar silica interface: Each gallium cell can exist in one of three states (Fig. 1) - a lowest-energy 'ground' state, higher-energy 'metallic' state, and a highest-energy, short-lived 'optically excited' state. The ground state possesses optical properties identical to those of solid $\alpha$-gallium, while the optically excited and metallic states possess those of liquid gallium. In each time

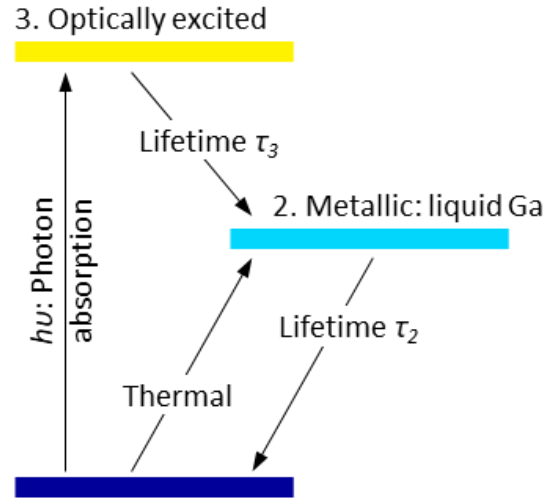

1. Ground: solid $\alpha-G a$

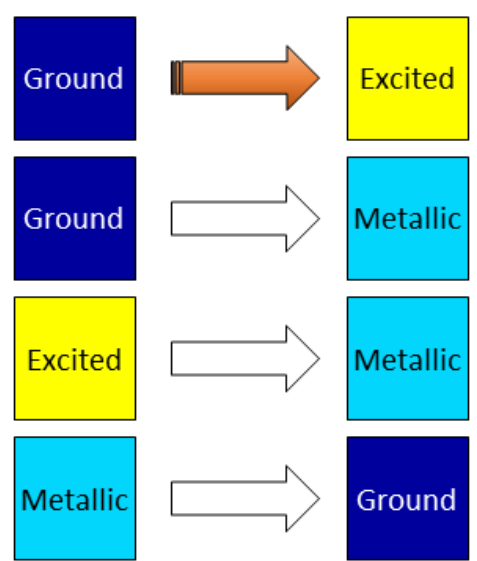

Absorption of light

Probabilistic, conditional on environment

Probabilistic

Probabilistic, conditional on environment

Fig. 1. Three-level system of gallium cell states and summary of transitions possible in each time step of the cellular automata model. Transitions that are conditional on the environment of a cell depend upon the phase state of neighboring cells and effective proximity of local temperature to the $29.8^{\circ} \mathrm{C}$ melting point of bulk a-Ga. 
step, each cell may or may not change its state according to a fixed set of rules dependent upon the incident photon flux, interface reflectivity (which is recalculated in each time step), rates of thermal and non-thermal excitation (from ground to metallic and excited states respectively), the lifetimes of the metallic and excited states, and the phase state of neighboring cells.

This CA model reproduces experimentally observed light-induced transition and relaxation dynamics of gallium over seven orders of excitation pulse duration magnitude from femtosecond to microsecond, and provides insight to the microscopic mechanisms of transition without recourse to deep understanding or analytical description of atomic dynamics, band structure, electromagnetics, thermodynamics or nanoscale energy conservation. It is found that transition and reflectivity dynamics are controlled predominantly by the relative values of optically-excited and metallic state lifetimes. These determine whether thermal or non-thermal mechanisms dominate and thereby whether the metallization of a-Ga is diffuse (in the short, fs-ps pulse regime - Fig. 2) or proceeds through the motion of a defined solid-liquid melt front (longer pulses). Interestingly, the CA model shows that neighborhood is essentially irrelevant in the short pulse excitation regime: i.e. at fs timescales there is no coupling between Ga (crystalline) cells, and each cell responds independently to the flux of incident photons.

(a)

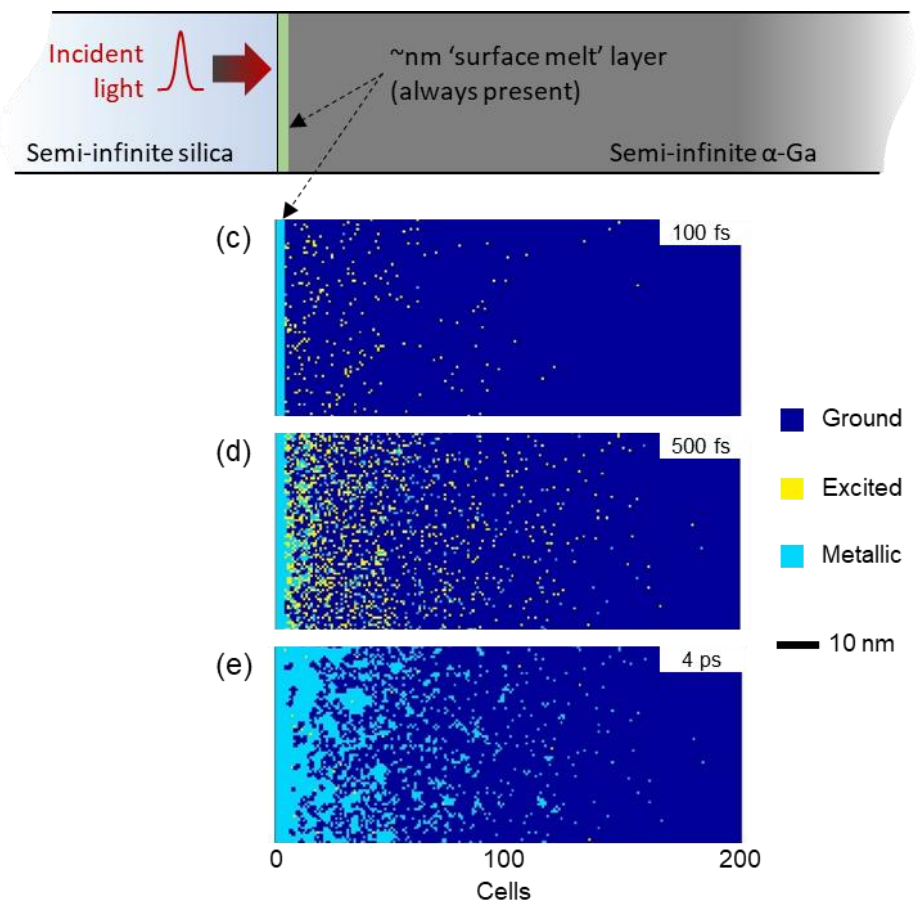

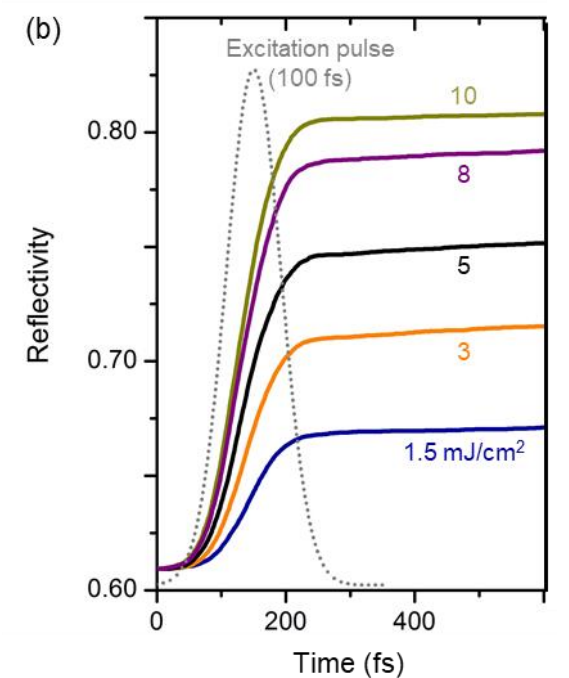

Fig. 2. CA model of Ga-silica interface reflectivity dynamics. (a) The model considers an infinite planar interface between semi-infinite silica and solid Ga, at which a nm liquid phase 'surface melt' layer persists even at temperatures below the bulk melting point of Ga and in the absence of optical excitation. (b) Interface reflectivity at $775 \mathrm{~nm}$ as a function of time during and immediately after $100 \mathrm{fs}$ pump pulses of varying fluence [as labelled]. The dashed grey line shows the Gaussian temporal profile of the excitation pulse [normalized amplitude - vertical axis scale does not apply]. (c, d, e) Representative CA maps of phase state distribution at selected time intervals: (c) during the early part of the pulse - a diffuse distribution of isolated cells are in the excited state; (d) immediately after the pulse - evolving balance of excited and metallic state cells distributed within the ground state bulk; (e) a few picoseconds after the pulse, an interval several times longer than the excited state lifetime - metallic state dynamics now dominate optical properties.

The cellular automata approach may be applied to a variety phase-change, nonlinear optical and active (nano)photonic systems - for example, the three-level model developed here for Ga may be adapted to $\mathrm{VO}_{2}$, where ultrafast transitions also appear to involve a transient electronically excited state. In an era of otherwise largely opaque computational modelling techniques, the cellular automata methodology has considerable heuristic value for the study of complex nonlinear optical processes and non-equilibrium behaviors in systems close to a transition point, which cannot be described analytically. 